\title{
Influencia de los recursos y capacidades en los resultados financieros y en la competitividad empresarial: una revisión de la literatura
}

Influence of resources and capabilities in finance performance and business competitiveness: a

literature review

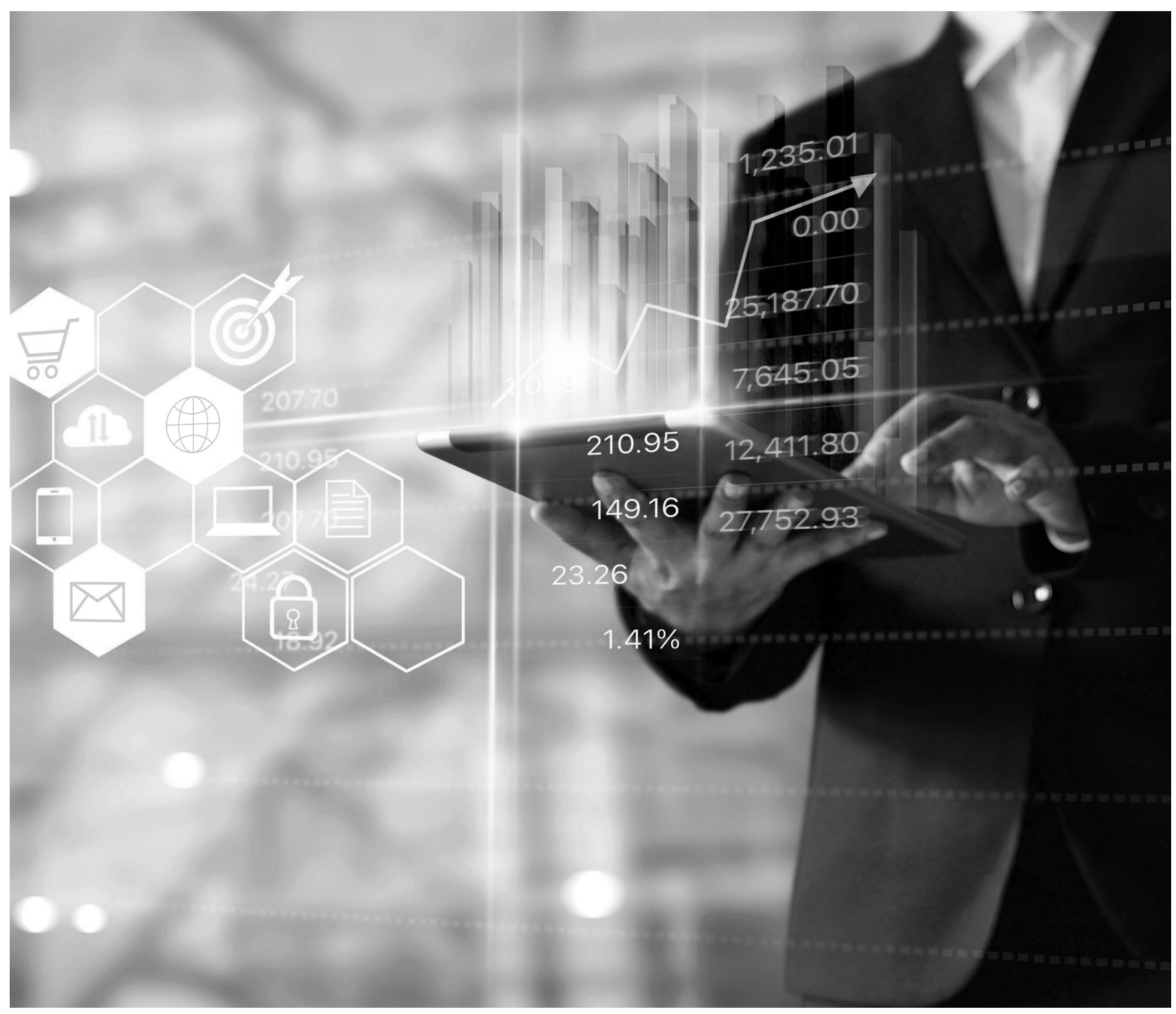




\title{
Influencia de los recursos y capacidades en los resultados financieros y en la competitividad empresarial: una revisión de la literatura ${ }^{1}$ Influence of resources and capabilities in finance performance and business competitiveness: a literature review
}

\author{
Julieth Katherin Acosta-Medina², Karen Rocío Plata-Gómez³ , David Esteban Puentes- \\ Garzón ${ }^{4}$, Martha Liliana Torres-Barreto ${ }^{5}$
}

Artículo recibido en octubre de 2018; artículo aceptado en noviembre de 2018.

Este artículo puede compartirse bajo la Licencia Creative Commons Atribución-NoComercial-Compartirlgual 4.0 Internacional y se referencia usando el siguiente formato: Acosta-Medina, J. K., Plata-Gómez, K. R., Puentes-Garzón, D. E. \& Torres-Barreto, M. L.

(2019). Influencia de los recursos y capacidades en los resultados financieros y en la competitividad empresarial: una revisión de literatura. I+D Revista de Investigaciones, 13 (1), 147-157. DOI: https://doi.org/10.33304/revinv.v13n1-2019013

\section{Resumen}

La estrategia empresarial es una herramienta que tiene como propósito obtener una ventaja competitiva sostenible a partir de las particularidades internas de las empresas, como pueden ser los recursos y las capacidades. Existen diversas formas de establecer dicha estrategia, una de estas tiene fundamento en la Teoría de Recursos y Capacidades, que intenta explicar la competitividad a través de los recursos y habilidades que posee la empresa o a los que puede acceder. Por esto, se realiza una revisión de literatura que consta de un análisis bibliométrico y de un análisis de la literatura consultada, logrando verificar la influencia de cierto tipo de recursos y capacidades sobre los resultados financieros y la competitividad empresarial, donde se destaca la combinación de las capacidades dinámicas junto a los recursos tangibles y/o humanos.

Palabras clave: recursos, capacidades, resultados financieros, competitividad, revisión literatura.

\footnotetext{
Abstract

The business strategy is a tool that has the aims to obtain a sustainable competitive advantage, from the internal

1. Artículo de revisión, con un enfoque cualitativo, resultado de un trabajo de grado para optar por el título de Ingeniería Industrial (en curso), perteneciente al área de Dirección Empresarial, desarrollado en el grupo de investigación Finance \& Management en la Universidad Industrial de Santander (Bucaramanga, Colombia). Dirección: Carrera 27 Calle 9, PBX: 6344000. Fecha de inicio: junio de 2018.

2. Estudiante de Ingeniería Industrial. Grupo de Investigación Finance \& Management, Universidad Industrial de Santander (Bucaramanga, Colombia). Dirección: Carrera 27 Calle 9, PBX: 6344000. ORCID ID: https://orcid.org/0000-0001-6910-069X. Correo electrónico institucional: Julieth. acosta1@correo.uis.edu.co.

3. Estudiante de Ingeniería Industrial. Grupo de Investigación Finance \& Management, Universidad Industrial de Santander (Bucaramanga, Colombia). Dirección: Carrera 27 Calle 9, PBX: 6344000. ORCID ID: https://orcid.org/0000-0001-8871-6377. Correo electrónico institucional: Karen.plata@correo.uis.edu.co. 4. Ingeniero Industrial y candidato a Magister en Ingeniería Industrial. Grupo de Investigación Ópalo, Universidad Industrial de Santander, (Bucaramanga, Colombia). Dirección Carrera 27 Calle 9, PBX: 6344000. ORCID ID: https://orcid.org/0000-0001-8178-2339. Correo electrónico institucional: david.puentes1@correo.uis.edu.co.

5. Doctora en Ciencias Económicas. Profesora e investigadora del Grupo de Investigación Finance \& Mangement, Universidad Industrial de Santander (Bucaramanga, Colombia). Dirección: Carrera 27 Calle 9, PBX: 6344000. ORCID ID: http://orcid.org/0000-0002-4388-5991 Correo electrónico institucional: mltorres@uis.edu.co.
} 
Julieth Katherin Acosta-Medina, Karen Rocío Plata-Gómez, David Esteban Puentes-Garzón, Martha Liliana Torres-Barreto Influencia de los recursos y capacidades en los resultados financieros y en la competitividad empresarial: una revisión de la literatura

peculiarities of companies, such as resources and capabilities. There are several ways to establish this strategy, one of these is based on the Theory of Resources and Capabilities, that attempts to explain competitiveness through the resources and skills that the firm possesses or can access. For this reason, it's done a literature review that consists of a bibliometric analysis and an analysis of the literature consulted, making it possible to verify the influence of certain types of resources and capabilities on financial performance and business competitiveness, where the combination of dynamic capabilities together with tangible and/or human resources.

Keywords: resources, capabilities, finance performance, competitiveness, literature review.

\section{Introducción}

"En 2017 el crecimiento económico mundial alcanzó el $3.0 \%$, porcentaje que representó una fuerte aceleración frente al $2.4 \%$ de 2016 y constituye la mayor tasa de crecimiento mundial registrada desde 2011" (United Nations, 2018). De acuerdo al Banco Mundial, la competitividad es el principal aspecto que las organizaciones deben tener, ya que permite fomentar el crecimiento económico en los países a partir de la generación de empleo, la inversión y el comercio, en respuesta a las oportunidades del mercado.

Es por esto que, actualmente, las organizaciones se enfrentan al reto de ser más competitivas ante un entorno cada vez más complejo y turbulento, de modo que están llamadas a desarrollar estrategias que busquen explotar al máximo sus características únicas, permitiéndoles mejorar sus resultados empresariales (Torres-Barreto \& Antolinez, 2017). Estas estrategias empresariales son un plan de acción que una organización, en interacción con su entorno, realiza para crear y mantener ventajas competitivas, entendiendo por ventaja competitiva una característica diferencial que una compañía tiene respecto a otras, lo que le confiere la capacidad para alcanzar unos rendimientos superiores de manera sostenible en el tiempo (Ariza, 2015).

Algunas de estas estrategias empresariales se desarrollan a partir de las particularidades internas de la empresa y tienen fundamento en la Teoría de Recursos y Capacidades (TRC), la cual busca reconocer el potencial de la empresa para establecer ventajas competitivas y mejorar sus resultados financieros (Barney, 1991) mediante la identificación y valoración de los recursos y habilidades que posee la empresa o a los que puede acceder; por tanto, los recursos y capacidades pueden ser la base de cualquier estrategia empresarial, y llegar a convertirse en la principal fortaleza de una organización (Suárez \& Ibarra, 2002).

En esta investigación se presenta una revisión de la literatura sobre la Teoría de Recursos y Capacidades y su incidencia en la competitividad empresarial, con el objetivo de identificar aquellos recursos y capacidades que influyen en el desempeño financiero para evaluarlos en empresas de diversos sectores y entornos.

\section{Teoría de Recursos y Capacidades}

La Teoría de Recursos y Capacidades se desarrolla en los años 80, partiendo del modelo de las cinco fuerzas de Porter (1980), herramienta que facilitaba entender la manera como el entorno competitivo externo influye en las empresas, pero era necesario hacer un análisis estratégico que permitiera investigar por qué, dentro de un mismo sector, se producen diferencias en el desempeño de las organizaciones. Por esta razón, se adopta una perspectiva de "adentro hacia afuera" o una perspectiva específica de la empresa con el estudio de las estrategias empresariales para crear ventajas competitivas sostenibles a partir de los recursos y las capacidades que poseen las empresas o que pueden adquirir, de esta perspectiva surge la Teoría de Recursos y Capacidades (Wernerfelt, 1986).

Esta teoría establece que los recursos y las capacidades son fuente de rentabilidad (Alvarez-Melgarejo \& Torres-Barreto, 2018b; Torres-Barreto, 2017), y a partir de ellos es posible formular estrategias a largo plazo que permitan explotar al máximo las características únicas de cada empresa, permitiéndoles mejorar sus resultados financieros (Suárez \& Ibarra, 2002). Si bien existen varios tipos de recursos y capacidades (AlvarezMelgarejo \& Torres-Barreto, 2018a) (ver Figura 1), los más importantes son aquellos durables, difíciles de identificar y comprender, imperfectamente transferibles, no replicables fácilmente y sobre los cuales la empresa posea control y posesión (Grant, 1991).

\section{Recursos}

Las entradas del proceso productivo, es decir activos físicos, procesos organizacionales, información y conocimiento, disponibles y controlables por la empresa, los que le permiten a la empresa establecer estrategias competitivas para mejorar su eficiencia y efectividad. Sin embargo, para generar una ventaja competitiva sostenible, estos recursos deben ser valiosos, raros, inimitables y no sustituibles, heterogéneos e inamovibles 
(Barney, 1991; Cuervo, 1993). De acuerdo a su naturaleza, se dividen en recursos tangibles, intangibles y humanos (Grant, 2010).

Recursos tangibles: Son los más fáciles de reconocer y evaluar, ya que se identifican y valoran en los estados financieros de la empresa (Grant, 1991). Entre los recursos tangibles se encuentran los recursos físicos y financieros, donde los primeros corresponden a terrenos, edificaciones, maquinaria y equipo, muebles de oficina e inventarios; mientras que los recursos financieros comprenden efectivo, capital, seguros y provisión de cartera (Alvarez-Melgarejo \& Torres-Barreto, 2018b; Grant, 2010).

Recursos intangibles: Comprenden patentes, derechos de autory de fabricación, secretos comerciales y recursos relacionados con la reputación como valor de la marca comercial, relaciones con clientes y proveedores (Grant, 1996, 2010) y cultura organizacional (Barney, 1991).

Recursos humanos: Compuestos por el talento humano que articula el organigrama de la empresa y su formación, entrenamiento, habilidades, esfuerzo, capacidad de comunicación y colaboración, motivación, know-how y experiencia (Barney, 1991).

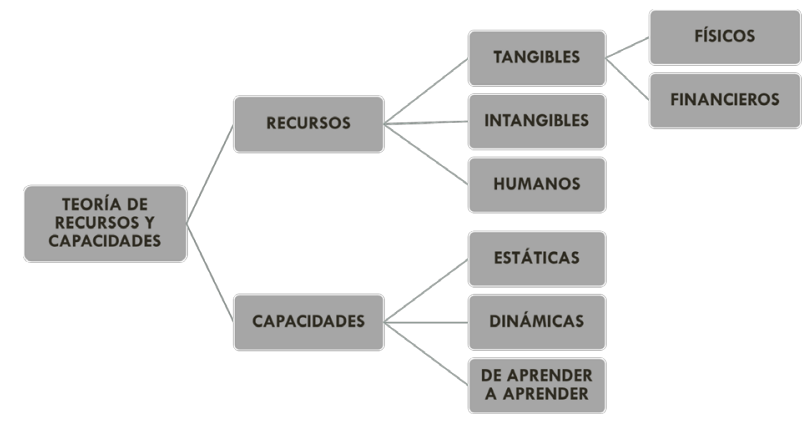

Figura 1. Tipos de recursos y capacidades. Fuente: Adaptado de Grant (2010) y Teece (1997).

\section{Capacidades}

Son un conjunto de recursos, conocimientos y habilidades que surgen del aprendizaje colectivo de la organización (Cuervo, 1993) como consecuencia de la coordinación y la cooperación entre el talento humano de la empresa y otros recursos (Suárez \& Ibarra, 2002); estas capacidades les confieren a las empresas una mayor aptitud respecto a su competencia, lo que las llevará a desarrollar ventajas competitivas sostenibles que se puedan traducir en mejores resultados financieros (Mazaira, Dopico \& González, 2005). En otras palabras, "los recursos son fuente de capacidades, pero las capacidades son la principal fuente de ventaja competitiva" (Grant, 1991).

Cabe destacar que las capacidades valiosas dependen del contexto, de la industria y del momento (Collis, 1994), pero generalmente se caracterizan por ser duraderas, tácitas, complejas, difíciles de imitar y de sustituir, raras, escasas, superiores a las de la competencia y deben contribuir de manera significativa a la generación de valor al consumidor, mejorando la competitividad (Mazaira et al., 2005). Teniendo en cuenta su importancia y complejidad, las capacidades están organizadas, tal como se muestra en la Figura 2, en estáticas, dinámicas y transformacionales (Teece, Pisano \& Shuen, 1997).

Capacidades estáticas: Corresponden a las actividades funcionales básicas de la empresa, como diseño de planta, logística de distribución y campañas de marketing, desarrolladas en áreas funcionales de la compañía (Teece, Pisano \& Shuen, 1997). Entre las capacidades estáticas se encuentran las capacidades de marketing funcionales y operativas (ver Figura 2). Las primeras son habilidades para adquirir, retener, expandir $y$, si es necesario, abandonar clientes; también hacen referencia a la capacidad de acceso a consumidores y a las destrezas de gestionar los productos actuales o desarrollar nuevos. Por otro lado, las capacidades de marketing operativas corresponden a la habilidad para implementar acciones de mercadeo relativas a precios, publicidad y promoción de ventas (Mazaira et al., 2005).

Capacidades dinámicas: Son las capacidades de la empresa que le permiten aprender, integrar, construir y reconfigurar las competencias internas y externas para abordar entornos que cambian rápidamente y así adaptarse, cambiar y renovarse a lo largo del tiempo (Teece, Pisano \& Shuen, 1997). Entre las capacidades dinámicas se encuentran las capacidades tecnológicas, de innovación, de absorción, estratégicas de marketing y de planeación (ver Figura 2).

Las capacidades tecnológicas son habilidades para hacer un uso efectivo de la tecnología a través de la inversión, producción, soporte y adaptación integral de las organizaciones a las tecnologías modernas, y su propósito es mejorar la eficiencia productiva (García, Pineda, Antonieta \& Vallejo, 2015). Por otra parte, las capacidades de innovación son habilidades cuyo objetivo es desarrollar nuevos productos, servicios y formas organizativas (García et al., 2015) a través de la transformación del conocimiento e ideas que pueden beneficiar a la compañía (Mathuramaytha, 2012). 
Asimismo, las capacidades de absorción permiten reconocer, asimilar, aplicar, combinar y utilizar, con fines comerciales, los recursos y la información procedente del exterior, siendo de gran importancia para la exportación (Zahra \& George, 2002). Por su parte, las capacidades estratégicas de marketing comprenden la capacidad de percepción del mercado que permiten identificar oportunidades de negocio y determinar los segmentos de mercados más propicios para la compañía (Mazaira et al., 2005). Finalmente, las capacidades de planeación les facilitan a las empresas adoptar diferentes tipos de estrategias para adaptarse a los cambios del entorno y sobresalir en un mercado altamente competitivo (Lau, Yam \& Tang, 2010).

Capacidades transformacionales: Comprende las ideas estratégicas que permiten a las empresas reconocer el valor intrínseco de otros recursos o desarrollar nuevas estrategias antes que sus competidores, aprendiendo a aprender y aprendiendo más rápido que la competencia (Collis, 1994).

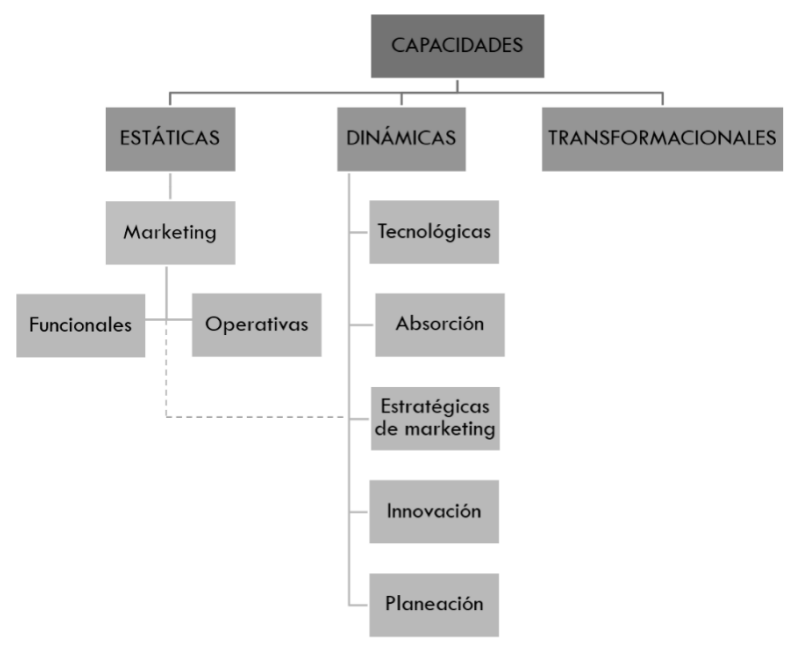

Figura 2. Tipos de capacidades. Fuente: Adaptado de David, Gary y Amy Shuen (1997) y David (1994).

\section{Resultados financieros}

Los resultados financieros son los que provienen de la realización de operaciones financieras y económicas, y se refieren a los niveles de liquidez, rentabilidad y riesgo que atraviesa una empresa en un período determinado. Estos resultados son consecuencia de la gestión que se haya efectuado en la entidad, por lo que son un buen indicador del desempeño corporativo; además, permiten la supervivencia y el desarrollo de las organizaciones. Entre ellos se destacan: ventas, exportaciones, rentabilidad y crecimiento de los ingresos.

\section{Competitividad empresarial}

Es la capacidad que posee una organización para incrementar o consolidar su presencia en el mercado aumentando de forma sostenida sus ventas y su rentabilidad, por lo tanto, la competitividad se relaciona con resultados cuantitativos como participación de mercado, número de sucursales/franquicias, número de clientes, etc. Las compañías se ven enfrentadas a desarrollarla, en particular, cuando se encuentran en un ambiente en el que necesitan competir para mantenerse y desarrollarse (Comisión Económica para América Latina y el Caribe [Cepal], 2017).

\section{Metodología}

\section{Tipo de estudio}

Se trata de una investigación con un enfoque cualitativo, ya que busca estudiar la influencia de los recursos $y$ las capacidades sobre los resultados financieros y la competitividad empresarial. El tipo de estudio es no experimental, aunque evalúa aspectos de la documentación científica, valorando alguna información que se puede extraer de los artículos cientíícos revisados; es de tipo longitudinal porque revisa la trazabilidad de la literatura desde los orígenes de la Teoría de Recursos y Capacidades.

\section{Procedimiento}

Para la presente revisión de literatura se realiza una búsqueda enfocada en la Teoría de Recursos y Capacidades y su influencia sobre los resultados financieros y la competitividad empresarial, haciendo uso de las bases de datos multidisciplinarias ISI Web of Science (WoS) y Scopus; la decisión de utilizar estas bases de datos se fundamenta en el importante reconocimiento internacional y el alto impacto de sus publicaciones (Aghaei et al., 2013).

Además, para identificar e incorporar los artículos bases y estructurales del tema de investigación, se utiliza la herramienta Tree of Science. Asimismo, para consolidar la información se revisan aquellas referencias bibliográficas que se citan en la literatura consultada y son importantes para la investigación, es decir, se realiza una búsqueda basada en el principio de bola de nieve. Posteriormente, los resultados derivados de las búsquedas son analizados utilizando el software Vantage Point. Finalmente, se hace un análisis en cuanto a los atributos de interés (recursos, capacidades, resultados y metodología empleada) abordados en algunos de los artículos más afines al enfoque de esta 
investigación, con el fin de reconocer la influencia de los recursos y capacidades en los resultados financieros y en la competitividad empresarial.

\section{Resultados}

En los resultados de esta investigación primero se presenta un análisis bibliométrico de los artículos consultados, seguido de un análisis de la revisión de literatura.

\section{Análisis bibliométrico}

En primera instancia, se propone una ecuación de búsqueda que incluye palabras clave que representan directamente los aspectos de mayor interés que se pretende abarcar en esta investigación. Por esto, se requiere la presencia de los términos "resource*" y "capabilit" a fin de encontrar artículos relacionados con la Teoría de Recursos y Capacidades; en este caso se utiliza la función asterisco ${ }^{*}$ ) con el objetivo incluir diferentes variaciones de estos términos en la búsqueda (como, "resource" o "resources" y"capability" o "capabilities").

A su vez, en la estructura de la ecuación se agrega como requisito que los artículos encontrados cuenten con alguno de los siguientes términos: "sale", "finance performance", "competitive advantage" o "export", haciendo referencia al aspecto de resultados financieros y competitividad empresarial. Por último, se emplean los términos "influence", "impact", "relation" y "effect" para relacionar resultados empresariales y TRC. En la Figura 3 se presentan las ecuaciones de búsqueda propuestas tanto en la base de datos Web of Science como en Scopus.

Web of Science: $T S=\left(\left(\right.\right.$ resource* AND capabilit* ${ }^{*}$ AND (sales OR "finance performance" OR "competitive advantage" OR export*) AND (influence OR impact OR relation OR effect))

Scopus: TITLE-ABS-KEY ((resource* AND capabilit*) AND (sales OR "finance performance" OR "competitive advantage" OR export*) AND (influence OR impact OR relation OR effect))

Figura 3. Primera ecuación de búsqueda. Fuente: Autores.

El total de resultados obtenidos en esta búsqueda es de 298 para WoS y de 149 para Scopus, entre estos se incluyen artículos, revisiones y memorias de eventos (proceedings). Esta búsqueda se realizó con todos los años disponibles en cada base de datos (del 2001 al 2018 para Web of Science y de 1976-2018 para Scopus), sin tener en cuenta restricciones de idioma, tipos de documentos o áreas de interés. A partir de los resultados se puede verificar que la TRC ha sido un tema que muestra una tendencia creciente en el número de artículos producidos con el paso de los años (ver Figura 4).

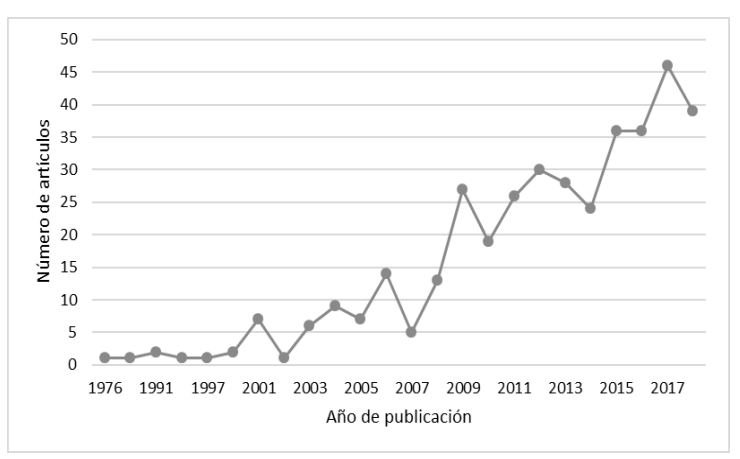

Figura 4. Publicaciones por año (total artículos encontrados). Fuente: Autores utilizando el software Vantange Point.

En este primer acercamiento, un gran número de publicaciones no se consideraron pertinentes para la investigación, por lo que se decidió refinar la búsqueda por áreas de estudio rehaciendo la ecuación y excluyendo aquellas que no tuvieran relación alguna con el tema de interés en la investigación (ver Figura 5).

\footnotetext{
Web of Science: TS $=(($ resource* AND capabilit*) AND (sales OR "finance performance" OR "competitive advantage" OR export*) AND (influence OR impact OR relation OR effect)) NOT $W C=$ (genetics heredity $O R$ hospitality leisure sport tourism $O R$ mathematical computational biology $O R$ engineering multidisciplinary OR multidisciplinary sciences OR environmental sciences $O R$ geosciences multidisciplinary OR psychology experimental $O R$ environmental studies $O R$ agriculture dairy animal science OR psychology multidisciplinary OR energy fuels OR engineering electrical electronic OR evolutionary biology OR water resources OR engineering environmental OR psychology applied OR ecology OR green sustainable science technology)

Scopus: TITLE-ABS-KEY (((resource* AND capabilit*) AND (sales OR "finance performance" OR "competitive advantage" OR export*) AND (influence OR impact OR relation OR effect) AND (exclude (subjarea, "envi") OR exclude (subjarea, "eart") OR exclude (subjarea, "phar") OR exclude (subjarea, "medi")) AND (exclude (subjarea, "agri") OR exclude (subjarea,"math") OR exclude (subjarea, "mult") OR exclude (subjarea, "arts")) AND (exclude (subjarea, "ener"))
}

Figura 5. Segunda ecuación de búsqueda. Fuente: Autores.

De los resultados obtenidos en esta nueva búsqueda se identificaron 38 artículos encontrados tanto en WoS como en Scopus, lo que dejó un total de 344 artículos. Adicionalmente, con la herramienta Tree of Science (ToS) se analizaron los 256 resultados de WoS (ToS solo permite leer archivos desde Web of Science), con el fin 
de identificar los artículos más relevantes. Valga anotar que esta herramienta muestra los artículos en forma de árbol, siendo la raíz los artículos seminales, el tronco los que dan estructura al tema y las hojas, los documentos más recientes. De este modo, se logran identificar artículos de gran importancia, de autores como Barney, Wernerfelt, Teece y Collins, pioneros en esta temática de investigación (ver Figura 6).
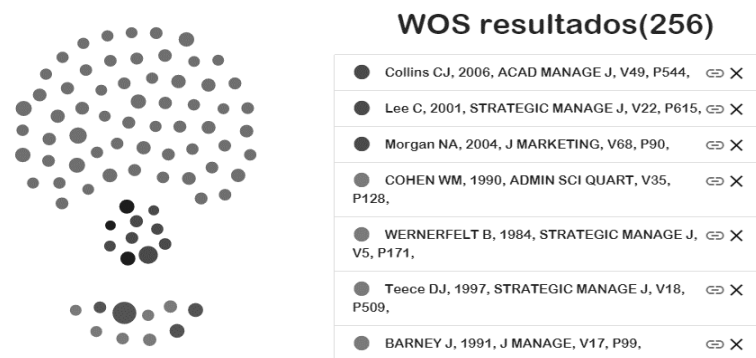

Figura 6. Resultados del análisis en ToS. Fuente: Autores utilizando la herramienta Tree of Science.

Después de analizar el total de documentos encontrados en la búsqueda y con ayuda de la herramienta ToS, se seleccionaron para estudio los 81 artículos considerados de mayor afinidad con el tema, los cuales constituyen el principal insumo para realizar el análisis presentado a continuación, que permite conocer la dinámica de publicación a nivel mundial considerando los campos bibliográficos de autores, países y años de publicación.

La Figura 7 muestra la publicación de artículos por países, donde se aprecia que Estados Unidos es el país con mayor número de producciones científicas en el sobre el tópico en cuestión; esto se debe, en parte, a que las primeras aproximaciones al tema fueron de origen estadounidense; adicionalmente, se puede ver que Colombia hace parte de los países que publicaron de 4 a 9 artículos. Asimismo, el gráfico de cantidad de publicaciones por año y país de la Figura 8 confirma que desde el año 1984 hasta el 2000 el tema de la Teoría de Recursos y Capacidades solo fue estudiado en los Estados Unidos, como se mencionó.

Por otra parte, para analizar la conexión entre autores se realiza el gráfico de aduna, el cual permite observar la manera como diferentes autores han colaborado entre sí para la producción de uno o más artículos científicos en esta temática. En la generación de esta aduna solo se incluyen colaboraciones entre más de tres autores, con el fin de mostrar las relaciones más relevantes (ver Figura 9).

Por ejemplo, en la aduna se puede observar que los autores Yam y Tang colaboraron en la producción de dos artículos junto con diferentes investigadores; asimismo, se ve la conexión en las producciones literarias de Takahashi e Inmyxai y Faroque.

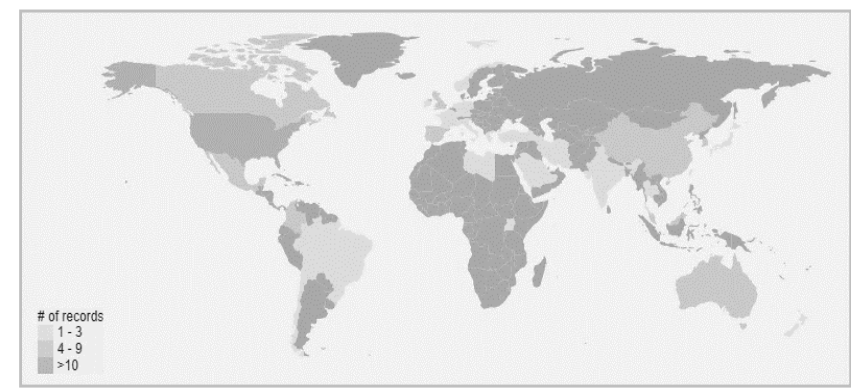

Figura 7. Países con mayor número de publicaciones. Fuente: Autores utilizando el software Vantange Point.

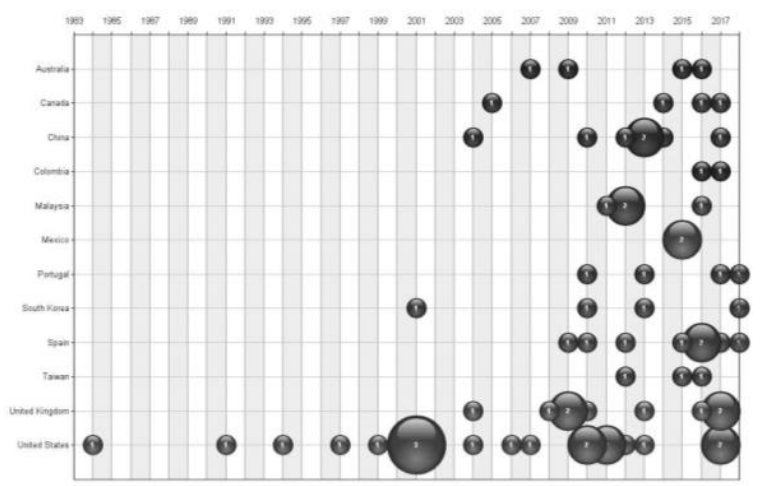

Figura 8. Cantidad de publicaciones por año en los países más representativos. Fuente: Autores utilizando el software Vantange Point.

\section{Análisis de la revisión de literatura}

Los estudios realizados por los diversos autores permitieron obtener diferentes resultados que confirman la influencia que tienen ciertos recursos y capacidades en los resultados financieros y la competitividad de las empresas. La Tabla 1 muestra las convenciones de los atributos usadas en la Tabla 2, la cual presenta una recopilación de los atributos de interés abordados en algunos de los artículos más afines al enfoque de esta investigación.

En la Tabla 2 se puede observar la forma como algunos autores han realizado diversos estudios basados en la Teoría de Recursos y Capacidades con el propósito de investigar la contribución relativa de los recursos tangibles y las capacidades dinámicas en función de medidas como rotación de ventas y rentabilidad (Kamasak, 2017), exportaciones (Morgan, Kaleka \& Katsikeas, 2004) y competitividad empresarial (Ismail, 
Rose, Uli \& Abdullah, 2012; Lee, Lee \& Pennings, 2001). Los datos recolectados en estas investigaciones fueron analizados en su mayoría mediante modelos regresivos, ya que estos permiten identificar la contribución única de cada variable independiente sobre la dependiente, característica que facilita relacionar directamente los resultados empresariales con la disponibilidad de recursos y capacidades.

Otros estudios analizan la manera como los recursos humanos tienen un impacto positivo en el rendimiento de la empresa; por ejemplo, la educación y las habilidades del propietario generalmente aumentan la fortaleza de la organización (Inmyxai \& Takahashi, 2009), así como el liderazgo estratégico del CEO (Lopez-Cabrales, Bornay-Barrachina \& Diaz-Fernandez, 2017). Del mismo modo, autores como Escandon, Arias y Salas, (2012) y
Mathuramaytha, (2012) han investigado la influencia del talento humano y las capacidades dinámicas sobre la competitividad empresarial, encontrando la existencia de una relación positiva entre estos.

Además, desde la perspectiva de las capacidades dinámicas se han realizado numerosas investigaciones, entre ellas los estudios de Bamel y Bamel (2018) y Lau et al. (2010) que establecen la vinculación entre los recursos de la organización y las capacidades dinámicas, comprobando que si estos se desarrollan en conjunto logran mejorar significativamente las ventas. Asimismo, González, López y De Castro (2009) han concluido que cuando la empresa desarrolla a la vez capacidades de absorción e innovación, obtiene un mayor beneficio operativo que si únicamente desarrollara la absorción (impacto negativo) o innovación de forma aislada.

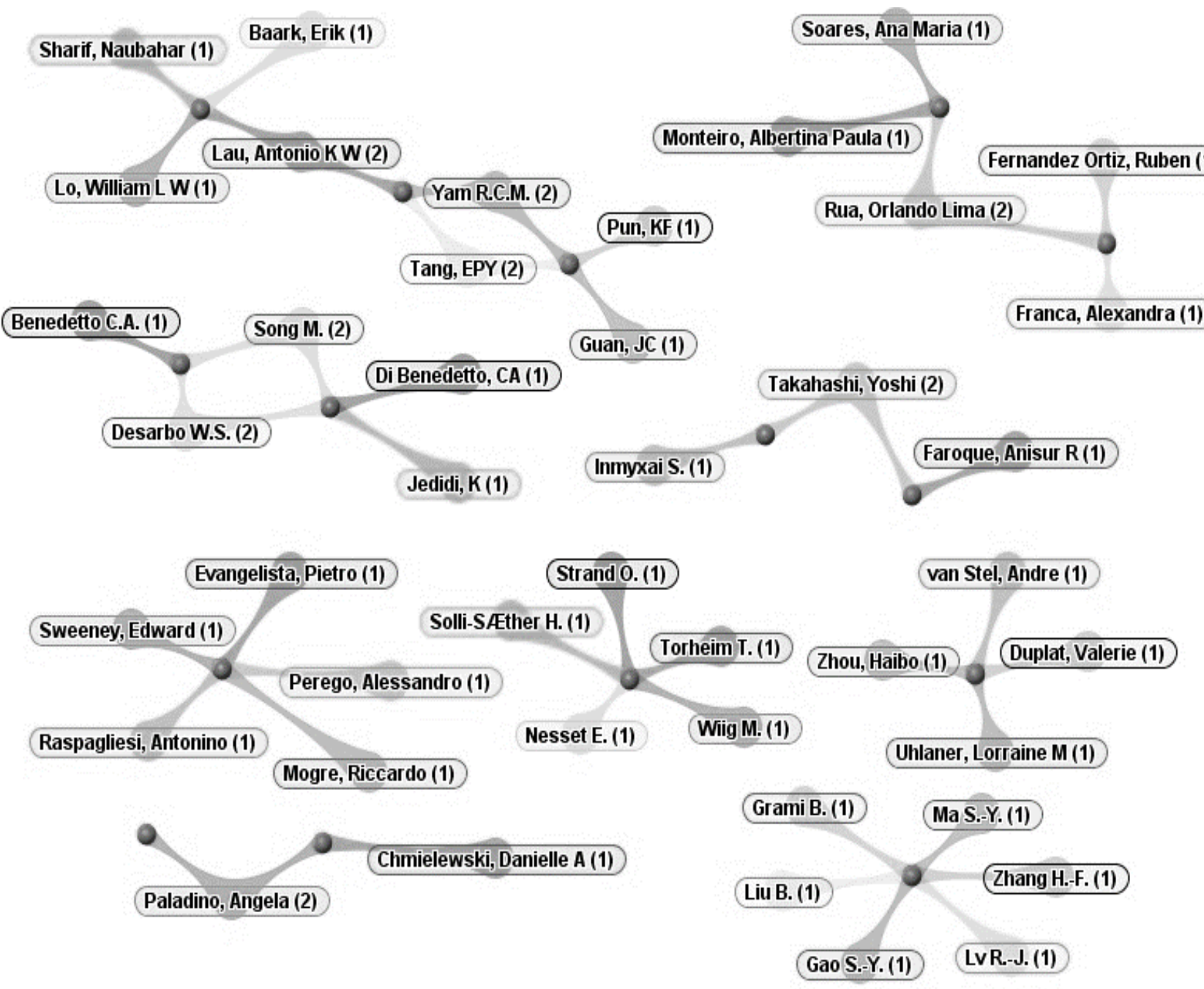

Figura 9. Aduna de autores para producciones científicas en colaboración. Fuente: Autores utilizando el software Vantage Point. 
Tabla 1

Convenciones de los atributos de interés para la presente investigación

\begin{tabular}{|c|c|}
\hline Convención & Atributo \\
\hline 1 & Recursos \\
\hline $1 \mathrm{a}$ & Tangibles \\
\hline $1 b$ & Intangibles \\
\hline $1 c$ & Humanos \\
\hline 2 & Capacidades \\
\hline $2 a$ & Estáticas \\
\hline $2 b$ & Dinámicas \\
\hline $2 c$ & Transformacionales \\
\hline 3 & Metodología \\
\hline $3 a$ & Regresión \\
\hline $3 b$ & Análisis factorial \\
\hline $3 c$ & Análisis de componentes principales (PCA) \\
\hline 4 & Resultados \\
\hline $4 a$ & Aumento en ventas \\
\hline $4 b$ & Aumento en exportaciones \\
\hline $4 c$ & Aumento en ingresos \\
\hline $4 d$ & Mejora en la competitividad \\
\hline
\end{tabular}

Fuente: Autores.

Tabla 2

Atributos encontrados en algunos de los artículos consultados

\begin{tabular}{|c|c|c|c|c|c|c|c|c|c|c|c|c|c|}
\hline \multirow[t]{2}{*}{ Artículo } & \multicolumn{3}{|c|}{1} & \multicolumn{3}{|c|}{2} & \multicolumn{3}{|c|}{3} & \multicolumn{4}{|c|}{4} \\
\hline & $1 \mathrm{a}$ & $1 \mathrm{~b}$ & 1c & $2 a$ & $2 b$ & $2 c$ & $3 a$ & $3 b$ & $3 c$ & $4 a$ & $4 \mathrm{~b}$ & $4 c$ & $4 d$ \\
\hline Lee et al., 2001 & $x$ & & & & $x$ & & $x$ & & & $x$ & & & \\
\hline Inmyxai \& Takahashi, 2009 & $x$ & $x$ & $x$ & $x$ & & & $x$ & & & $x$ & & $x$ & \\
\hline Morgan et al., 2004 & $x$ & & & & $x$ & & $x$ & $x$ & & & $x$ & $x$ & \\
\hline Bamel \& Bamel, 2018 & & $x$ & & & $x$ & & $x$ & & & & & & $x$ \\
\hline Lopez-Cabrales et al., 2017 & & & $x$ & & $x$ & & $x$ & $x$ & & & & & \\
\hline González et al., 2009 & & & & & $x$ & & $x$ & & & $x$ & & & \\
\hline Kamasak, 2017 & $x$ & $x$ & & $x$ & $x$ & & $x$ & & & $x$ & & & \\
\hline Faroque \& Takahashi, 2015 & $x$ & & $x$ & & & & $x$ & & $x$ & & $x$ & & \\
\hline Escandon et al., 2012 & & & $x$ & & $x$ & & $x$ & $x$ & $x$ & & & & $x$ \\
\hline Ismail et al., 2012 & $x$ & $x$ & $x$ & & $x$ & & $x$ & & & & & & $x$ \\
\hline Lau et al., 2010 & & & & & $x$ & & $x$ & & & $x$ & & & \\
\hline Mathuramaytha, 2012 & & & $x$ & & $x$ & & $x$ & & & & & & $x$ \\
\hline
\end{tabular}

Fuente: Autores. 


\section{Conclusiones}

A partir de los resultados obtenidos en las distintas investigaciones consultadas es posible verificar el cumplimiento de la Teoría de Recursos y Capacidades en el ámbito empresarial. Asimismo, de acuerdo a la revisión de literatura realizada en este trabajo, se logran identificar algunas combinaciones específicas de recursos y capacidades que, desarrolladas en conjunto, permiten a las empresas obtener mayores rendimientos financieros y mejorar la competitividad. Entre estas combinaciones se destacan: las capacidades dinámicas que junto con los recursos humanos y/o los recursos tangibles facilitan la obtención de mayores ventas e ingresos y mejoran la competitividad. Con base en lo anterior, las empresas tienen la opción de formular diversas estrategias basadas en la combinación de dichos recursos y capacidades que, en conjunto, permiten obtener mejores resultados empresariales que sí se desarrollarán individualmente. Este trabajo permite tener un panorama más claro sobre la Teoría de Recursos y Capacidades y de la manera como a partir de esta, las empresas en el mercado actual pueden desarrollar estrategias dirigidas al mejoramiento de los diversos aspectos que conforman el rendimiento empresarial, obteniendo ventajas competitivas frente a la competencia. Esta investigación, además, busca aportar a la literatura en sobre el tema y ser punto de partida para futuras investigaciones donde se busque evaluar si estos recursos y capacidades tienen la misma influencia en los diferentes sectores económicos a nivel regional o nacional.

\section{Referencias}

Aghaei Chadegani, A., Salehi, H., Yunus, M., Farhadi, H., Fooladi, M., Farhadi, M. \& Ale Ebrahim, N. (2013). A Comparison between Two Main Academic Literature Collections: Web of Science and Scopus Databases. Asian Social Science, 9(5), 18-26. https://doi. org/10.5539/ass.v9n5p18>

Alvarez-Melgarejo, M. \& Torres-Barreto, M. L. (2018a). Can resources act as capabilities foundations? A bibliometric analysis. Revista UIS Ingenierías, 17(2), 185-200. https://doi.org/http://orcid.org/0000-00024388-5991

Alvarez-Melgarejo, M. \& Torres-Barreto, M. L. (2018b). Recursos y capacidades: factores que mejoran la capacidad de absorción. I+D Revista de Investigaciones, $12(2), 51-58$.

Ariza, H. M. (2015). Revisitando estrategias de sostenibilidad de las empresas a través de una visión sistémica empresarial. I+D Revista de Investigaciones, 5(1), 23-42.

Bamel, U. K. \& Bamel, N. (2018). Organizational resources,
KM process capability and strategic flexibility: a dynamic resource-capability perspective. Journal of Knowledge Management. https://doi.org/10.1108/ JKM-10-2017-0460

Barney, J. (1991). Firm Resorces and sustained Competitive Advantage. Journal of Management, 17, 99-120.

Collis, D. J. (1994). Research Note: How Valuable are Organizational Capabilities? Strategic Management Journal, 15(S1), 143-152.

Comisión Económica para América Latina y el Caribe. (2017). Estudio Económico de América Latina y el Caribe 2017: la dinámica del ciclo económico actual y los desafíos de política para dinamizar la inversión y el crecimiento. Santiago de Chile.

Cuervo García, A. (1993). El papel de la empresa en la competitividad. Papeles de Economía Española, (56), 363-377.

Escandon, D. M., Arias, A. \& Salas, J. A. (2012). Recursos y capacidades que inciden en el desempeño de los subsectores económicos colombianos 2003-2004. Ingeniería y Competitividad, 14(2), 53-67.

Faroque, A. R. \& Takahashi, Y. (2015). Export marketing assistance and early internationalizing firm performance Does export commitment matter? Asia Pacific Journal Of Marketing And Logistics, 27(3), 421443. https://doi.org/10.1108/APJML-03-2014-0045

García Velázquez, A., Pineda Domínguez, D., Antonieta, M. \& Vallejo, A. (2015). Las capacidades tecnológicas para la innovación en empresas de manufactura. Universidad \& Empresa, 17(29), 257-278. https://doi. org/10.12804/rev.univ.empresa.29.2015.11

González, J., López Sáez, P. \& De Castro, G. (2009). La Influencia de las Capacidades Dinámicas sobre los Resultados Financieros de la Empresa Pedro LÓPEZ SÁEZ, 19, 105-128.

Grant, R. M. (1991). The Resource-Based Theory of Competitive Advantage: Implications for Strategy Formulation. California Management Review, 33(3), 114-135. https://doi.org/10.2307/41166664

Grant, R. M. (1996). Dirección estratégica. Conceptos, técnicas y aplicaciones. Madrid: Civitas.

Grant, R. M. (2010). Contemporary Strategy Analysis. Barcelona.

Inmyxai, S.\&Takahashi, Y. (2009). Firm resources and business performance in the Lao PDR: Implications for SMEs in the LDC context. Journal of Indian Business Research, 1, 163-187.https://doi.org/10.1108/17554190911005345

Ismail, A. I., Rose, R. C., Uli, J. \& Abdullah, H. (2012). The relationship between organisational resources, capabilities, systems and Competitive Advantage. Asian Academy of Management Journal, 17(1), 151173. https://doi.org/10.3923/ibm.2012.176.186

Kamasak, R. (2017). The contribution of tangible and intangible resources, and capabilities to 
Julieth Katherin Acosta-Medina, Karen Rocío Plata-Gómez, David Esteban Puentes-Garzón, Martha Liliana Torres-Barreto Influencia de los recursos y capacidades en los resultados financieros y en la competitividad empresarial: una revisión de la literatura

a firm's profitability and market performance. European Journal of Management and Business Economics, 26(2), 252-275. https://doi.org/10.1108/ EJMBE-07-2017-015

Lau, A. K. W., Yam, R. C. M. \& Tang, E. P. Y. (2010). The impact of technological innovation capabilities on innovation performance: An empirical study in Hong Kong. Journal of Science and Technology Policy in China, 1(2), 163-186. https://doi.org/10.1108/17585521011059893

Lee, C., Lee, K. \& Pennings, J. M. (2001). Internal capabilities, external networks, and performance: A study on technology-based ventures. Strategic Management Journal. https://doi.org/10.1002/smj.181

Lopez-Cabrales, A., Bornay-Barrachina, M. \& Diaz-Fernandez, M. (2017). Leadership and dynamic capabilities: the role of HR systems. Personnel Review, 46(2), 255-276. https:// doi.org/10.1108/PR-05-2015-0146

Mathuramaytha, C. (2012). The Impacts of intellectual capital on innovative capability: Building the sustain competitive advantage on a resource-based perspective of Thailand industrials. International Business Management, 6(4), 451-457. https://doi. org/10.3923/ibm.2012.451.457

Mazaira Castro, A., Dopico Parada, A. \& González Vázquez, E. (2005). Incidencia del grado de orientación al mercado de las organizaciones empresariales en el desarrollo de las capacidades estratégicas de marketing. Revista Europea de Dirección y Economía de La Empresa, 14(3), 181-208.

Morgan, N. A., Kaleka, A.\&Katsikeas, C. S. (2004). Antecedents of export venture performance: $A$ theoretical model and empirical assessment. Journal of Marketing, 68, 90-108.

Porter, M.E. (1980), Competitive Strategy: Techniques for Analyzing Industries and Competitors, The Free Press, New York, NY.

Suárez, J. \& Ibarra, S. (2002). La teoría de los recursos y las capacidades. Un enfoque actual en la estrategia empresarial. Anales de Estudios Económicos y Empresariales, 15, 63-89.

Teece, D. J., Pisano, G. \& Shuen, A. (1997). Dynamic Capabilities and Strategic Management. StraStrategic Management Journal, 18(7), 509-533.

Torres-Barreto, M. (2017). Product innovations and R \& $D$ public funding: How to handle heteroscedasticity and autocorrelation. I+D Revista de Investigaciones, 9(1), 138-145.

Torres-Barreto, M. L. \& Antolinez, D. F. (2017). Exploring the boosting potential of intellectual resources and capabilities on firm's competitiveness. Espacios, 38(31). Recuperado de http://www.revistaespacios. com/a17v38n31/a17v38n31p35.pdf

United Nations. (2018). Situación y perspectivas de la economía mundial 2018. Recuperado de https:// www.un.org/development/desa/dpad/publication/ situacion-y-perspectivas-de-la-economiamundial-2018/

Wernerfelt, B. (1986). A Resource-based View of the Firm. Strategic Management Journal, 5(2), 171-180.

Zahra, S. A. \& George, G. (2002). Absorptive capacity: A review, reconceptualization, and extension. Academy of Management Review, 27(2), 185-203. https://doi. org/https://doi.org/10.5465/AMR.2002.6587995 